\title{
A Statistical Parametric Analysis on Various Filters Applied on Different Noises for MRI and USG Images
}

\author{
Manasi Rana \\ Research Scholar \\ 2, Kuthiala Colony, Jodhamal Road, civil Lines, \\ Hoshiarpur-146001,Punjab,India
}

\author{
Jasdeep Kaur \\ Assistant Professor \\ 93 Kirpa Nagar, Naraingarh \\ chheharta, Amritsar-143005, Punjab, India
}

\begin{abstract}
This paper presents the comprehensive study of the various filters and their comparative analysis using statistical parameters in digital image processing. We have simulated the various statistical parameters and viewed their results using plots. We have done their comparative analysis with the help of MATLAB simulation to ease the selection of best filter for a specific noise introduced in MRI and USG image.
\end{abstract}

\section{Keywords}

Statistical Parameters, noise, filters, MRI and USG image.

\section{INTRODUCTION}

Suppression of noise efficiently in an image is a very important. Here the detection of the noise patterns which affect the USG and MRI image and to apply the Spatial filters to remove those noises. The common noises found in these images are speckle noise, salt and pepper noise and Gaussian noise. These images come across these noises from background and through equipment [1]. The noise can be of any type, high or low leading to the degradation of the image quality. The Spatial Filters are categorized according to their performance judged by Statistical analysis to find the best suitable Spatial Filter for a particular noise. The various statistical parameters used are MSE, NAE, NCC, max diff and PSNR. In the next few sections we have discussed about the various noises, filters and the statistical parameters for an input MRI and USG image. Section II deals with the statistical model of the proposed work done throughout the project in brief. Section III discusses about various noises used, followed by statistical parameters in section IV and section $\mathrm{V}$ contains experimental results for de noised images passed through filters.

\section{PROPOSED STATISTICAL MODE}

Although research has already been done on few of these measures at quite advance level, we have proposed a simple statistical model for experimental work. The proposed statistical model consists of the following steps:

1. Adding noise to input image, $I(x, y)$ : In this step noise is added to input image. Here three basis noises are added to input image differently, namely: Speckle, Salt and pepper and Gaussian noise.

2. Passing of noisy image through various filters : The noisy image is passed through various filters namely: Maximum filter, Minimum filter, Midpoint filter, Median filter, Harmonic filter, Contra harmonic mean filter, Arithmetic Mean filter, Alpha mean filter, Alpha trimmed mean filter and de noised images are obtained.
3.Statistical parameters for Comparing de noised images: Various statistical parameters used are Maximum Difference(MD), Normalized Absolute Error(NAE), Normalized Cross Correlation(NCC), Structural Content(SC), Average Difference(AD), Mean square Error(MSE), Peak Signal-to-Noise Ratio(PSNR). These parameters compared the various de noised images. The compared values for these parameters are given by comparison tables and plots. The best filter is retained through those values for all the noises.

\section{VARIOUS NOISES USED}

The various noises used for converting an input noiseless image into noisy image are: Speckle, Salt and pepper and Gaussian noise.

A. Speckle Noise: Speckle noise is defined to have a granular pattern. Speckle is the result of the diffused scattering, which occurs when an ultrasound pulse randomly interferes with small particles or objects comparable to the sound waves. In this, the ratio of variance and mean is constant over a wide region which is also a property of Rayleigh distribution [2].

$$
F(g)=\frac{g^{\alpha-1}}{(\alpha-1) ! \alpha^{\alpha}} e^{-\frac{g}{\alpha}}
$$

B. Salt and pepper noise: The corrupted pixel values are set alternatively to the maximum or to the minimum value, giving the image a salt and pepper like appearance as salt looks like white(one) and pepper looks as black(zero) for binary ones. For an 8 bit image, value of pepper noise is 0 (minimum) and for salt noise 255(maximum) [3]. Image with salt and pepper noise.

C. Gaussian Noise: Gaussian noise is statistical noise which has probability density function equal to that of the normal distribution [4], also known as the Gaussian distribution. A special case is white Gaussian noise, in which values at any pair of times are statistically independent and are uncorrelated [5].

$$
F(g)=\frac{1}{\sqrt{2 \pi \sigma * \sigma}} e^{-\left(g-m^{2} / 2 \sigma^{2}\right)}
$$

\section{Various Filters Used}

Each noise is passed through a particular set of filters to get the resultant output image.

A. Max Filter: The max filter, also known as the 100th percentile filter, replaces it with the maximum value. Max filter is usually used to identify the brightest pixel gray value of an image [5]. The brightest pixel becomes the new pixel value at the center of the window. This filter is normally considered best for the removal of salt and pepper noise. 


$$
F(x, y)=\max (s, t) \epsilon s_{x y}\{g(s, t)\}
$$

B. Min Filter: The min filter, also known as the zeroth percentile filter, replaces it with lowest value [5]. Min filter is used to identify the darkest pixel gray level value and usually used to remove salt noise from the images.

$$
F(x, y)=\min (s, t) € s_{x y}\{g(s, t)\}
$$

C. Midpoint Filter: The midpoint filter [5] calculates average of highest and lowest pixel values in a window by combining ordered statistics and averaging them into one filter. It is used to reduce Gaussian and uniform noise in images.

$$
\begin{aligned}
F(x, y)=\max (s, t) & \epsilon s_{x y}\{g(s, t)\} \\
& +\min (s, t) \epsilon s_{x y}\{g(s, t)\}
\end{aligned}
$$

D. Harmonic Mean Filter: In harmonic mean filter, the color value of each pixel is replaced with harmonic mean of pixels in the neighborhood. The harmonic mean filter [16] has another variation of the arithmetic mean filter and is useful for images with Gaussian or salt noise.

$$
H=\frac{n}{\frac{1}{X 1}+\frac{1}{X 2} \cdots \cdots \cdots \cdots \frac{1}{X n}}
$$

E. Contra Harmonic Mean Filter: Contra Harmonic Mean is a function complementary to the harmonic mean [4]. Image with salt noise can be filtered using negative value of $R$, whereas image with pepper noise can be filtered with positive values of $R$.

$$
\mathrm{C}(\mathrm{X} 1, \mathrm{X} 2 \ldots . \mathrm{Xn})=\frac{X 1^{2}+X 2^{2}+\cdots+\frac{X n^{2}}{n}}{X 1+X 2+\cdots \frac{X n}{n}}
$$

F. Alpha Mean Filter: It forms a hybrid of mean and median filters. It discards the elements at the start and end of ordered set and calculates its average or mean value [5].

G. Median Filter: Median Filter is also kind of non linear filter which preserves edges whilst removing noise. It works by selecting middle pixel from the ordered set of values within ' $\mathrm{m} \times \mathrm{n}$ ' neighborhood around the reference pixel [5].

$$
M(u, v)=\operatorname{median}\{I(x, y)(x, y) \in w F\}
$$

H. Alpha Trimmed Mean Filter: In this case an average of the pixel values closest to the median, after the ' $D$ ' lowest and ' $\mathrm{D}$ ' highest values in ordered set have been removed [5]. The filter behaves as a regular arithmetic mean filter. For $D=(m n-1) / 2$ it is equivalent to the median filter.

$$
\mathrm{F}(\mathrm{x}, \mathrm{y})=\frac{1}{\mathrm{mn}-2 \mathrm{D}} \sum_{(\mathrm{r}, \mathrm{c}) € \mathrm{~W}} \mathrm{~g}(\mathrm{r}, \mathrm{c})
$$

\section{STATISTICAL PARAMETERS}

Statistics is defined as the study of the collection, organization, analysis, and interpretation of data. Here we will study various types of statistical measures with respect to image processing and simulated all of these.
A. Maximum Filter: It differentiates between original and output image after the removal of noise. It must be least for the best filter.

$$
M D=\max |x(i, j)-y(i, j)|
$$

B. Normalized Absolute Error: It is used to determine the outcome image to the input image. Its value must be closer to zero for better results.

$$
N A E=\frac{\sum_{i=1}^{M} \sum_{j=1}^{N}(x(i, j)-y(i, j))^{2}}{\sum_{i=1}^{M} \sum_{j=1}^{N}(x(i, j))^{2}}
$$

C. Structural Content: Structured content is more related to human perception than Mean Square Error values. If value comes out to be 1 means the original image and the resultant images are identical.

$$
S C=\frac{\sum_{i=1}^{M} \sum_{j=1}^{N}(y(i, j))^{2}}{\sum_{i=1}^{M} \sum_{j=1}^{N}(x(i, j))^{2}}
$$

D. Average Difference: Also called mean difference is defined as the value obtained of absolute difference of the two images. The average difference should be least means more similar are the images hence better is the filter.

$$
A D=\frac{1}{M N} \sum_{i=1}^{M} \sum_{j=1}^{N}(x(i, j)-y(i, j))
$$

E. Mean Square Error: Also called root mean squared error (RMSE) denotes mean square error of two images $\mathrm{m} x \mathrm{n}$ as $\mathrm{I}(\mathrm{i}, \mathrm{j})$ and $\mathrm{K}(\mathrm{i}, \mathrm{j})$ where one of the image is considered as the noisy approximation of the other image[6].

$$
M S E=\frac{1}{m n} \sum_{i=0}^{m-1} \sum_{j=0}^{n-1}[I(i, j)-K(i, j)]^{2}
$$

F. Peak Signal-to- Noise Ratio: The lower value of PSNR represents that the image reconstructed is of lower quality and vice-versa. It is mostly defined by using means square error as shown in the above equation [6].

$$
P S N R_{d b}=10 \log _{10} \frac{M A X_{I}^{2}}{M S E}
$$

\section{EXPERIMENTAL RESULTS}

The results are obtained by introducing different noises to original image forming noisy images. Each noisy image is further passed through various filters to de noise them. Then we compare them by calculating various statistical parameters explained above [7]. The values of these parameters are compared to find the best possible filter for a particular kind of noise including plots.

\section{A. Results for Speckle Noise:}
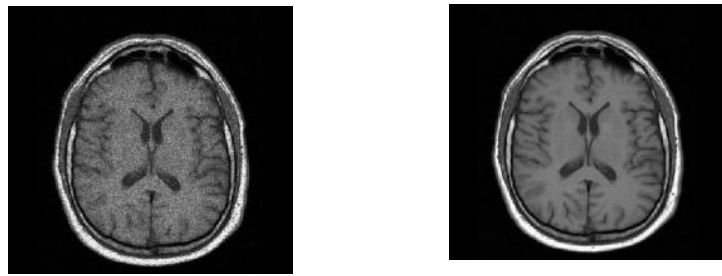


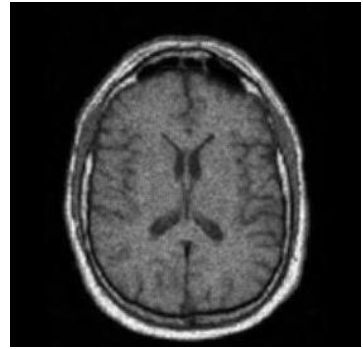

(C)

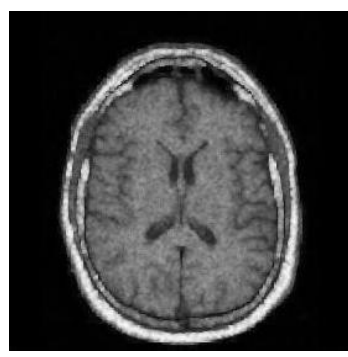

(E)

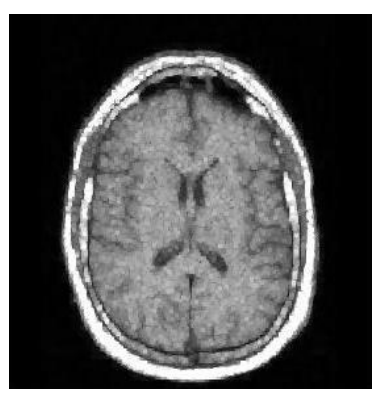

(G)

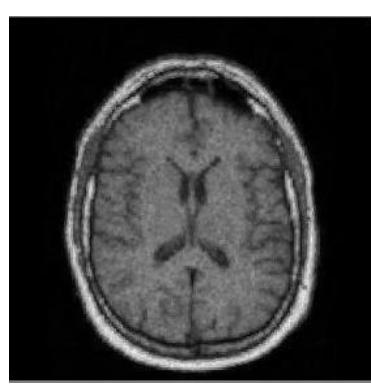

(I)

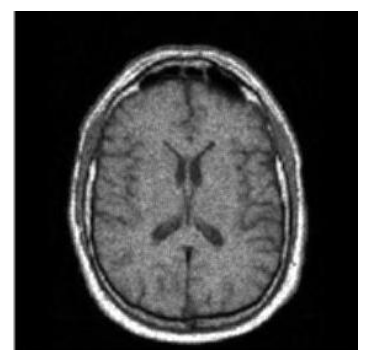

(D)

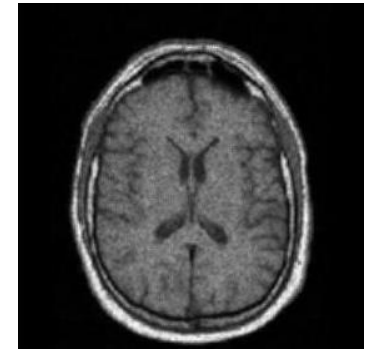

(F)

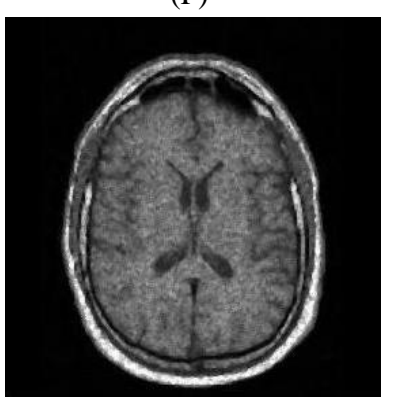

(H)

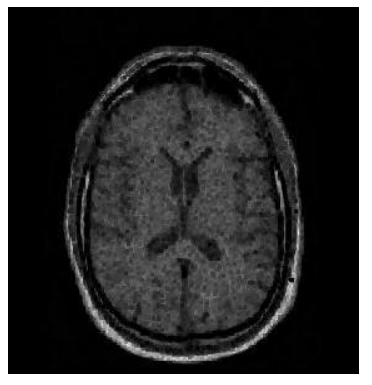

(J)
(A) Original input MRI image (B) Output image with speckle noise (C) Alpha trimmed mean filter image for speckle noise (D) Arithmetic mean filter image for speckle noise (E) Contra harmonic mean filter for speckle noise (F) Harmonic filter for speckle noise (G) Maximum filter image for speckle noise (H) Medium filter for speckle noise (I) Midpoint filter for speckle noise (J) Minimum filter image for speckle noise.

\begin{tabular}{|c|c|c|c|c|c|c|c|}
\hline & $\begin{array}{l}\text { Max } \\
\text { differ } \\
\text { ence }\end{array}$ & $\begin{array}{c}\text { NA } \\
\text { E }\end{array}$ & $\begin{array}{c}\mathrm{NC} \\
\mathrm{C}\end{array}$ & $\begin{array}{c}\text { Struct } \\
\text { ural } \\
\text { conte } \\
\text { nt }\end{array}$ & $\begin{array}{c}\text { Avera } \\
\text { ge } \\
\text { differ } \\
\text { ence }\end{array}$ & MSE & $\begin{array}{c}\text { PSN } \\
\mathrm{R}\end{array}$ \\
\hline $\begin{array}{l}\text { Alpha } \\
\text { trimm } \\
\text { ed } \\
\text { mean } \\
\text { filter }\end{array}$ & $\begin{array}{l}- \\
22.82 \\
98\end{array}$ & $\begin{array}{l}0.3 \\
895\end{array}$ & $\begin{array}{l}1.3 \\
895\end{array}$ & $\begin{array}{l}0.517 \\
9\end{array}$ & $\begin{array}{l}- \\
22.82 \\
98\end{array}$ & $\begin{array}{l}521.1 \\
978\end{array}$ & $\begin{array}{l}20.9 \\
608\end{array}$ \\
\hline $\begin{array}{l}\text { Arith } \\
\text { metic } \\
\text { mean } \\
\text { filter }\end{array}$ & $\begin{array}{l}- \\
24.87 \\
9\end{array}$ & $\begin{array}{l}0.4 \\
127\end{array}$ & $\begin{array}{l}1.4 \\
127\end{array}$ & $\begin{array}{l}0.501 \\
1\end{array}$ & $\begin{array}{l}- \\
24.18 \\
79\end{array}$ & $\begin{array}{l}585.0 \\
532\end{array}$ & $\begin{array}{l}20.4 \\
588\end{array}$ \\
\hline $\begin{array}{l}\text { Contr } \\
\text { a } \\
\text { harmo } \\
\text { nic } \\
\text { mean } \\
\text { filter }\end{array}$ & $\begin{array}{l}- \\
21.94 \\
20\end{array}$ & $\begin{array}{l}0.3 \\
744\end{array}$ & $\begin{array}{l}1.3 \\
744\end{array}$ & $\begin{array}{l}0.529 \\
4\end{array}$ & $\begin{array}{l}- \\
21.94 \\
20\end{array}$ & $\begin{array}{l}481.4 \\
528\end{array}$ & $\begin{array}{l}21.3 \\
053\end{array}$ \\
\hline $\begin{array}{l}\text { Harm } \\
\text { onic } \\
\text { mean } \\
\text { filter }\end{array}$ & $\begin{array}{l}- \\
27.17 \\
42\end{array}$ & $\begin{array}{l}0.4 \\
253\end{array}$ & $\begin{array}{l}1.4 \\
253\end{array}$ & $\begin{array}{l}0.466 \\
8\end{array}$ & $\begin{array}{l}- \\
27.17 \\
42\end{array}$ & $\begin{array}{l}738.4 \\
372\end{array}$ & $\begin{array}{l}19.4 \\
477\end{array}$ \\
\hline $\begin{array}{l}\text { Maxi } \\
\text { mum } \\
\text { filter }\end{array}$ & $\begin{array}{l}- \\
18.98 \\
15\end{array}$ & $\begin{array}{l}0.4 \\
042\end{array}$ & $\begin{array}{l}1.4 \\
042\end{array}$ & $\begin{array}{l}0.570 \\
6\end{array}$ & $\begin{array}{l}- \\
18.95 \\
15\end{array}$ & $\begin{array}{l}360.2 \\
973\end{array}$ & $\begin{array}{l}22.5 \\
642\end{array}$ \\
\hline $\begin{array}{l}\text { Media } \\
\mathrm{n} \\
\text { filter }\end{array}$ & $\begin{array}{l}- \\
24.92 \\
36\end{array}$ & $\begin{array}{l}0.5 \\
295\end{array}$ & $\begin{array}{l}1.5 \\
295\end{array}$ & $\begin{array}{l}0.492 \\
3\end{array}$ & $\begin{array}{l}- \\
24.92 \\
36\end{array}$ & $\begin{array}{l}621.1 \\
839\end{array}$ & $\begin{array}{l}20.1 \\
986\end{array}$ \\
\hline $\begin{array}{l}\text { Midp } \\
\text { oint } \\
\text { filter }\end{array}$ & $\begin{array}{l}- \\
23.69 \\
08\end{array}$ & $\begin{array}{l}0.3 \\
975\end{array}$ & $\begin{array}{l}1.3 \\
975\end{array}$ & $\begin{array}{l}0.507 \\
1\end{array}$ & $\begin{array}{l}- \\
23.69 \\
08\end{array}$ & $\begin{array}{l}561.2 \\
531\end{array}$ & $\begin{array}{l}20.6 \\
392\end{array}$ \\
\hline $\begin{array}{l}\text { Mini } \\
\text { mum } \\
\text { filter }\end{array}$ & $\begin{array}{l}- \\
31.03 \\
18\end{array}$ & $\begin{array}{l}0.2 \\
564\end{array}$ & $\begin{array}{l}1.2 \\
564\end{array}$ & $\begin{array}{l}0.427 \\
5\end{array}$ & $\begin{array}{l}- \\
31.03 \\
18\end{array}$ & $\begin{array}{l}962.9 \\
736\end{array}$ & $\begin{array}{l}18.2 \\
947\end{array}$ \\
\hline
\end{tabular}

Table 1: Comparison of Statistical parameters for Speckle noise in MRI image 


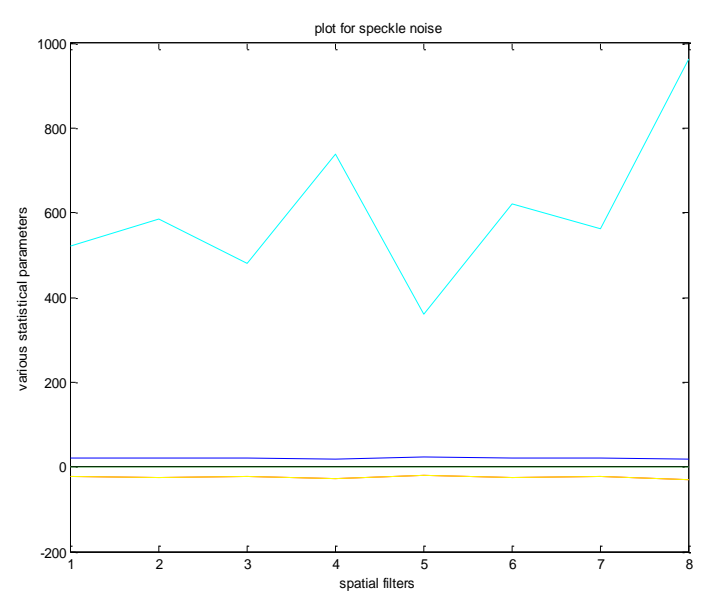

Graph 1: Plot of parameters for Speckle noise in MRI image

Maximum filter forms the best filter from the results for Speckle noise in MRI image.

B. Results for Salt and pepper noise:

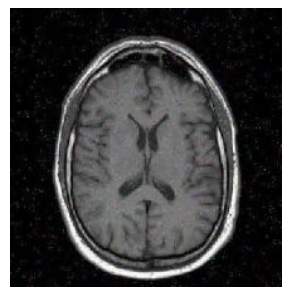

(A)

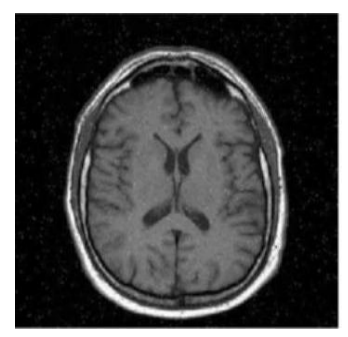

(C)

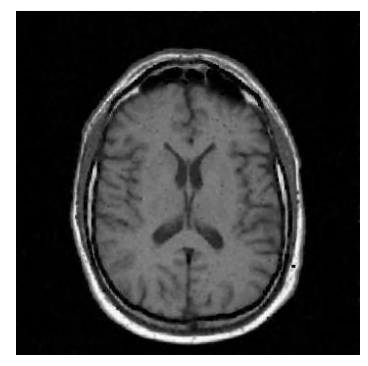

(E)

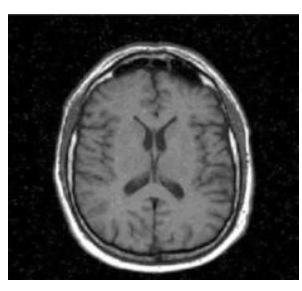

(B)

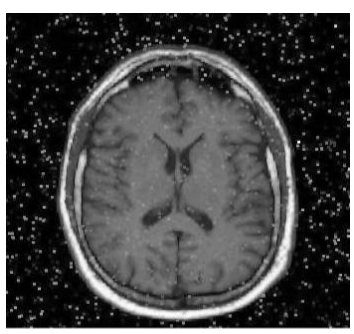

(D)

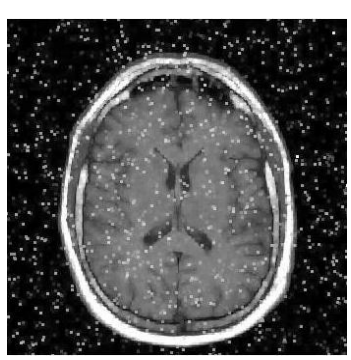

(F)

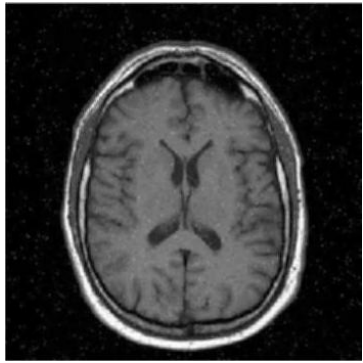

(G)

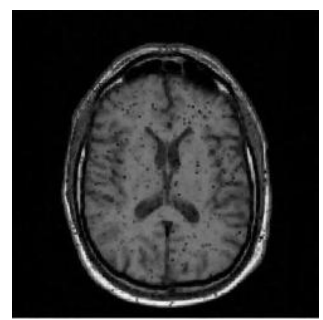

(I)

(A) Salt and pepper noise in MRI image (B) Alpha trimmed filtered image for salt and pepper noise (C) Arithmetic mean filtered image for salt and pepper noise (D) Contra harmonic mean filtered image for Salt and pepper noise (E) Harmonic mean filtered image for salt and pepper noise (F) Maximum filtered image for salt and pepper noise(G) Median filtered image for salt and pepper noise (H) Midpoint Filtered image for salt and pepper noise (I) Minimum Filtered image for salt and pepper noise.

\begin{tabular}{|c|c|c|c|c|c|c|c|}
\hline & $\begin{array}{l}\text { Max } \\
\text { diff }\end{array}$ & $\begin{array}{l}\text { NA } \\
\text { E }\end{array}$ & $\begin{array}{l}\mathrm{NC} \\
\mathrm{C}\end{array}$ & $\begin{array}{l}\text { Struc } \\
\text { tural } \\
\text { conte } \\
\text { nt }\end{array}$ & $\begin{array}{l}\text { Aver } \\
\text { age } \\
\text { differ } \\
\text { ence }\end{array}$ & MSE & $\begin{array}{l}\text { PSN } \\
\text { R }\end{array}$ \\
\hline $\begin{array}{l}\text { Alpha } \\
\text { trimm } \\
\text { ed } \\
\text { mean } \\
\text { filter }\end{array}$ & $\begin{array}{l}- \\
22.2 \\
98\end{array}$ & $\begin{array}{l}0.3 \\
895\end{array}$ & $\begin{array}{l}1.3 \\
895\end{array}$ & $\begin{array}{l}0.517 \\
9\end{array}$ & $\begin{array}{l}- \\
22.82 \\
98\end{array}$ & $\begin{array}{l}521 . \\
1978\end{array}$ & $\begin{array}{l}20.9 \\
608\end{array}$ \\
\hline $\begin{array}{l}\text { Arith } \\
\text { metic } \\
\text { mean } \\
\text { filter }\end{array}$ & $\begin{array}{l}- \\
43.8 \\
176\end{array}$ & $\begin{array}{l}0.7 \\
476\end{array}$ & $\begin{array}{l}1.7 \\
476\end{array}$ & $\begin{array}{l}0.327 \\
4\end{array}$ & $\begin{array}{l}- \\
43.81 \\
76\end{array}$ & $\begin{array}{l}1.92 \\
00 \\
e+03\end{array}$ & $\begin{array}{l}15.2 \\
978\end{array}$ \\
\hline $\begin{array}{l}\text { Contr } \\
\text { a } \\
\text { harmo } \\
\text { nic }\end{array}$ & $\begin{array}{l}- \\
40.1 \\
376\end{array}$ & $\begin{array}{l}0.6 \\
849\end{array}$ & $\begin{array}{l}1.6 \\
849\end{array}$ & $\begin{array}{l}0.352 \\
3\end{array}$ & $\begin{array}{l}- \\
40.13 \\
76\end{array}$ & $\begin{array}{l}1.61 \\
10 \\
e+03\end{array}$ & $\begin{array}{l}16.0 \\
598\end{array}$ \\
\hline
\end{tabular}




\begin{tabular}{|c|c|c|c|c|c|c|c|}
\hline $\begin{array}{l}\text { mean } \\
\text { filter }\end{array}$ & & & & & & & \\
\hline $\begin{array}{l}\text { Harm } \\
\text { onic } \\
\text { mean } \\
\text { filter }\end{array}$ & $\begin{array}{l}- \\
46.8 \\
521\end{array}$ & $\begin{array}{l}0.7 \\
994\end{array}$ & $\begin{array}{l}1.7 \\
994\end{array}$ & $\begin{array}{l}0.308 \\
8\end{array}$ & $\begin{array}{l}- \\
46.85 \\
21\end{array}$ & $\begin{array}{l}521 . \\
1978\end{array}$ & $\begin{array}{l}20.9 \\
608\end{array}$ \\
\hline $\begin{array}{l}\text { Maxi } \\
\text { mum } \\
\text { filter }\end{array}$ & $\begin{array}{l}- \\
37.5 \\
307\end{array}$ & $\begin{array}{l}0.6 \\
404\end{array}$ & $\begin{array}{l}1.6 \\
404\end{array}$ & $\begin{array}{l}0.371 \\
6\end{array}$ & $\begin{array}{l}- \\
37.53 \\
07\end{array}$ & $\begin{array}{l}1.40 \\
85 \\
e+03\end{array}$ & $\begin{array}{l}16.6 \\
431\end{array}$ \\
\hline $\begin{array}{l}\text { Media } \\
\mathrm{n} \\
\text { filter }\end{array}$ & $\begin{array}{l}- \\
43.8 \\
176\end{array}$ & $\begin{array}{l}0.7 \\
476\end{array}$ & $\begin{array}{l}1.7 \\
476\end{array}$ & $\begin{array}{l}0.327 \\
4\end{array}$ & $\begin{array}{l}- \\
43.81 \\
76\end{array}$ & $\begin{array}{l}521 . \\
1978\end{array}$ & $\begin{array}{l}20.9 \\
608\end{array}$ \\
\hline $\begin{array}{l}\text { Midp } \\
\text { oint } \\
\text { filter }\end{array}$ & $\begin{array}{l}- \\
42.1 \\
192\end{array}$ & $\begin{array}{l}0.7 \\
187\end{array}$ & $\begin{array}{l}1.7 \\
187\end{array}$ & $\begin{array}{l}0.338 \\
5\end{array}$ & $\begin{array}{l}- \\
42.11 \\
92\end{array}$ & $\begin{array}{l}1.77 \\
40 \\
\text { e+03 }\end{array}$ & $\begin{array}{l}15.6 \\
412\end{array}$ \\
\hline $\begin{array}{l}\text { Mini } \\
\text { mum } \\
\text { filter }\end{array}$ & $\begin{array}{l}- \\
49.3 \\
612\end{array}$ & $\begin{array}{l}0.8 \\
422\end{array}$ & $\begin{array}{l}1.8 \\
422\end{array}$ & $\begin{array}{l}0.297 \\
4\end{array}$ & $\begin{array}{l}- \\
49.36 \\
12\end{array}$ & $\begin{array}{l}2.43 \\
65 \\
e+03\end{array}$ & $\begin{array}{l}14.2 \\
631\end{array}$ \\
\hline
\end{tabular}

Table 2: Comparison of Statistical parameters for Salt and pepper noise in MRI image.

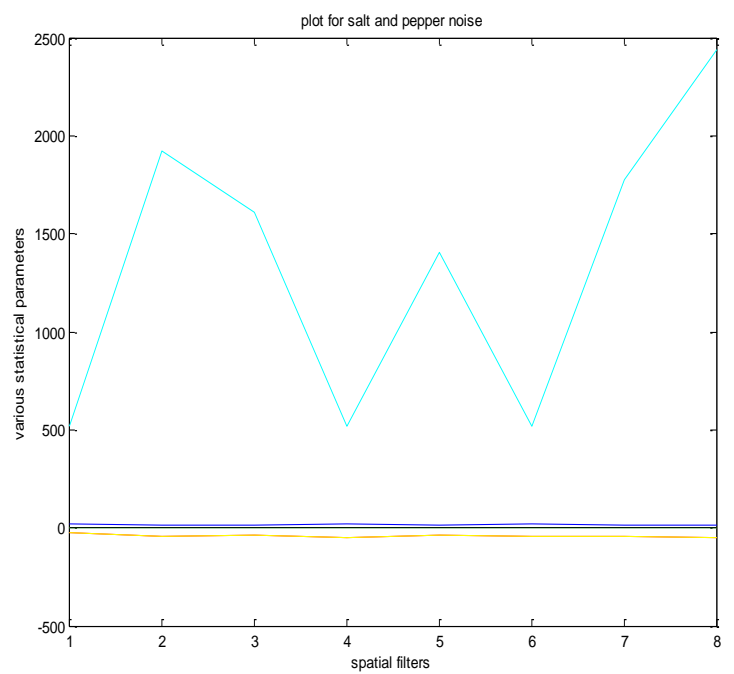

Graph 2: Plot of parameters for Salt and pepper noise in MRI image.

For MRI image it was found that alpha trimmed filter is the best filter according to the results obtained.

\section{Results for Gaussian noise:}

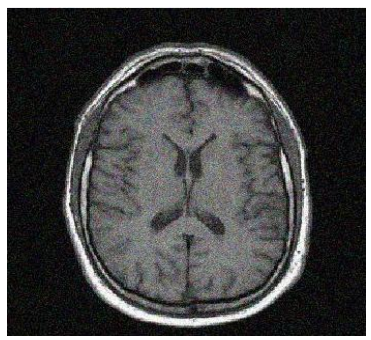

(A)

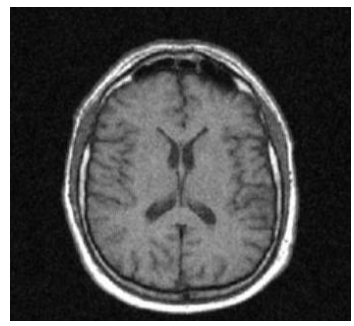

(C)

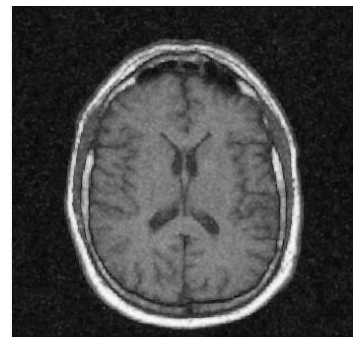

(E)

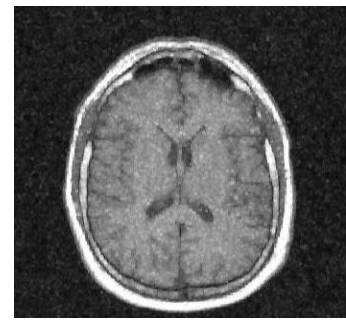

(G)

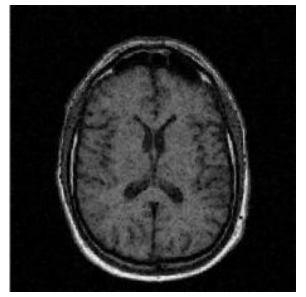

(I)

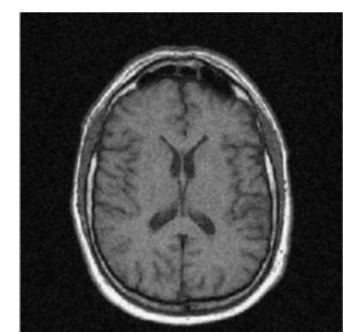

(B)

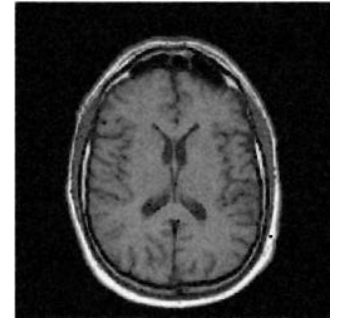

(D)

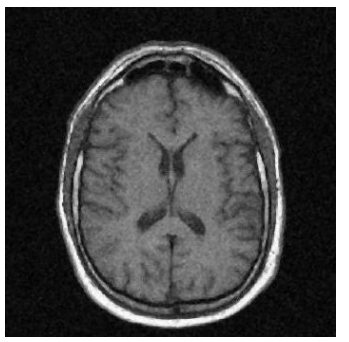

(F)

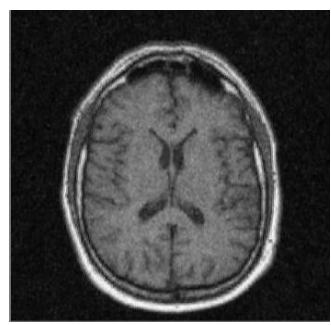

(H)
(A) Image with Gaussian noise of MRI (B) Alpha trimmed filtered image for Gaussian noise (C) Arithmetic mean filtered image for gaussian noisenoise (D) Harmonic mean filtered image for Gaussian noise (E) Contra harmonic mean filtered image for Gaussian noise (F) Maximum filtered image for Gaussian noise (G) Median filtered image for gaussian noise (H) Midpoint filtered image for Gaussian noise (I) Minimum filtered image for Gaussian noise 


\begin{tabular}{|c|c|c|c|c|c|c|c|}
\hline & $\begin{array}{l}\text { Max } \\
\text { diff }\end{array}$ & $\begin{array}{l}\text { NA } \\
\text { E }\end{array}$ & $\begin{array}{l}\mathrm{NC} \\
\mathrm{C}\end{array}$ & $\begin{array}{l}\text { Struct } \\
\text { ural } \\
\text { conte } \\
\text { nt }\end{array}$ & $\begin{array}{l}\text { Avera } \\
\text { ge } \\
\text { differ } \\
\text { ence }\end{array}$ & MSE & $\begin{array}{l}\text { PSN } \\
\mathrm{R}\end{array}$ \\
\hline $\begin{array}{l}\text { Alpha } \\
\text { trimm } \\
\text { ed } \\
\text { mean } \\
\text { filter }\end{array}$ & $\begin{array}{l}- \\
38.1 \\
102\end{array}$ & $\begin{array}{l}0.6 \\
503\end{array}$ & $\begin{array}{l}1.6 \\
503\end{array}$ & $\begin{array}{l}0.367 \\
2\end{array}$ & $\begin{array}{l}- \\
38.11 \\
02\end{array}$ & $\begin{array}{l}1.452 \\
4 \\
\text { e+03 }\end{array}$ & $\begin{array}{l}16.5 \\
100\end{array}$ \\
\hline $\begin{array}{l}\text { Arith } \\
\text { metic } \\
\text { mean } \\
\text { filter }\end{array}$ & $\begin{array}{l}- \\
40.0 \\
947\end{array}$ & $\begin{array}{l}0.6 \\
841\end{array}$ & $\begin{array}{l}1.6 \\
841\end{array}$ & $\begin{array}{l}0.352 \\
6\end{array}$ & $\begin{array}{l}- \\
40.09 \\
47\end{array}$ & $\begin{array}{l}1.607 \\
6 \\
\text { e+03 }\end{array}$ & $\begin{array}{l}16.0 \\
691\end{array}$ \\
\hline $\begin{array}{l}\text { Contr } \\
\text { a } \\
\text { harmo } \\
\text { nic } \\
\text { mean } \\
\text { filter }\end{array}$ & $\begin{array}{l}- \\
35.8 \\
658\end{array}$ & $\begin{array}{l}0.6 \\
120\end{array}$ & $\begin{array}{l}1.6 \\
120\end{array}$ & $\begin{array}{l}0.384 \\
8\end{array}$ & $\begin{array}{l}- \\
35.86 \\
58\end{array}$ & $\begin{array}{l}1.286 \\
\mathrm{e}+03\end{array}$ & $\begin{array}{l}17.0 \\
372\end{array}$ \\
\hline $\begin{array}{l}\text { Harm } \\
\text { onic } \\
\text { mean } \\
\text { filter }\end{array}$ & $\begin{array}{l}- \\
45.1 \\
120\end{array}$ & $\begin{array}{l}0.7 \\
697\end{array}$ & $\begin{array}{l}1.7 \\
697\end{array}$ & $\begin{array}{l}0.319 \\
3\end{array}$ & $\begin{array}{l}- \\
45.11 \\
20\end{array}$ & $\begin{array}{l}2.035 \\
1 \\
\text { e+03 }\end{array}$ & $\begin{array}{l}15.0 \\
450\end{array}$ \\
\hline $\begin{array}{l}\text { Maxi } \\
\text { mum } \\
\text { filter }\end{array}$ & $\begin{array}{l}- \\
30.2 \\
679\end{array}$ & $\begin{array}{l}0.5 \\
165\end{array}$ & $\begin{array}{l}1.5 \\
165\end{array}$ & $\begin{array}{l}0.434 \\
9\end{array}$ & $\begin{array}{l}- \\
30.26 \\
97\end{array}$ & $\begin{array}{l}916.1 \\
468\end{array}$ & $\begin{array}{l}18.5 \\
12\end{array}$ \\
\hline $\begin{array}{l}\text { Media } \\
\mathrm{n} \text { filter }\end{array}$ & $\begin{array}{l}- \\
40.9 \\
856\end{array}$ & $\begin{array}{l}0.6 \\
993\end{array}$ & $\begin{array}{l}1.6 \\
993\end{array}$ & $\begin{array}{l}0.346 \\
3\end{array}$ & $\begin{array}{l}- \\
40.98 \\
56\end{array}$ & $\begin{array}{l}1.679 \\
8 \\
e+03\end{array}$ & $\begin{array}{l}15.8 \\
782\end{array}$ \\
\hline $\begin{array}{l}\text { Midpo } \\
\text { int } \\
\text { filter }\end{array}$ & $\begin{array}{l}- \\
39.0 \\
472\end{array}$ & $\begin{array}{l}0.6 \\
662\end{array}$ & $\begin{array}{l}1.6 \\
662\end{array}$ & $\begin{array}{l}0.360 \\
2\end{array}$ & $\begin{array}{l}- \\
39.04 \\
72\end{array}$ & $\begin{array}{l}1.524 \\
7 \\
\text { e+03 }\end{array}$ & $\begin{array}{l}16.2 \\
990\end{array}$ \\
\hline $\begin{array}{l}\text { Mini } \\
\text { mum } \\
\text { filter }\end{array}$ & $\begin{array}{l}- \\
50.0 \\
220\end{array}$ & $\begin{array}{l}0.8 \\
535\end{array}$ & $\begin{array}{l}1.8 \\
535\end{array}$ & $\begin{array}{l}0.291 \\
1\end{array}$ & $\begin{array}{l}- \\
50.02 \\
20\end{array}$ & $\begin{array}{l}1.524 \\
7 \\
\text { e+03 }\end{array}$ & $\begin{array}{l}14.5 \\
896\end{array}$ \\
\hline
\end{tabular}

Table 3: Comparison of Statistical parameters for Gaussian noise in MRI image

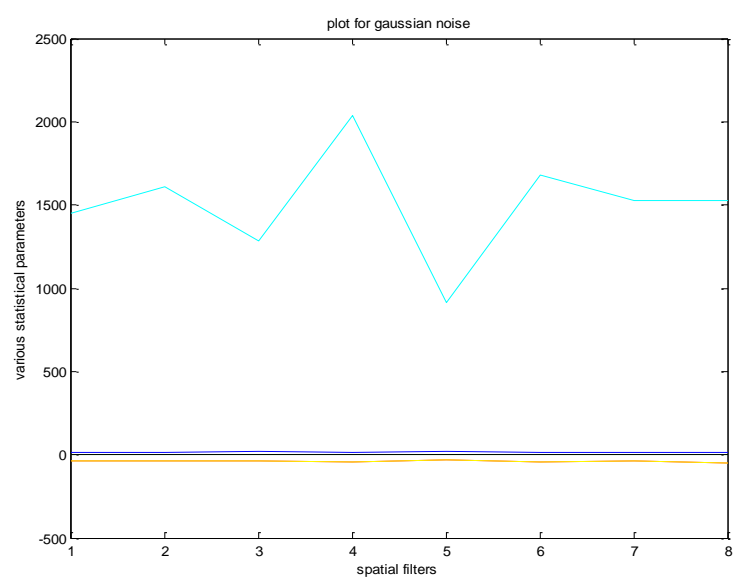

Graph 3: Plot of parameters for Gaussian noise in MRI image.

Comparing all the images of various filters for Gaussian noise in MRI image Maximum filter is the best filter.

D. Results for speckle noise in USG image:

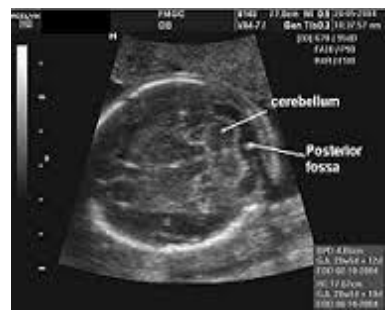

(A)

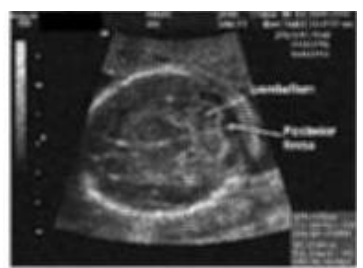

(C)

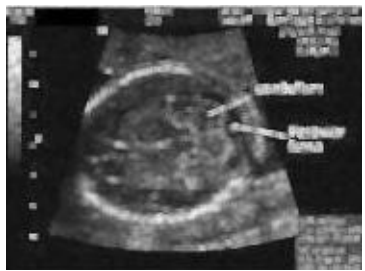

(E)

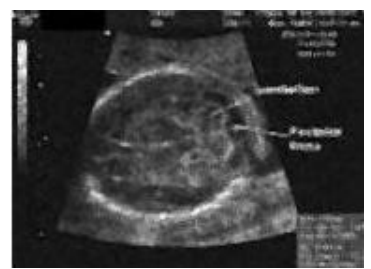

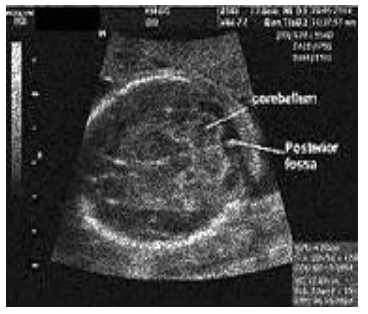

(B)

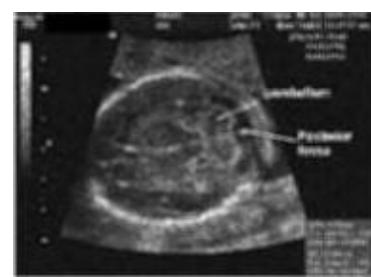

(D)

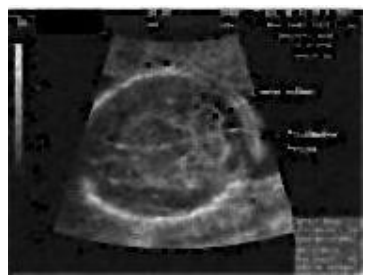

(F)

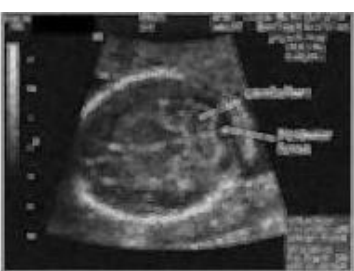


(G)

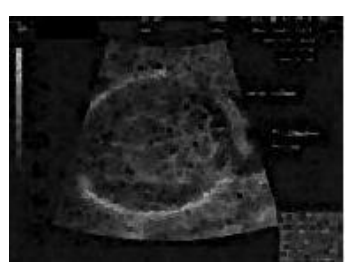

(H)

(I)

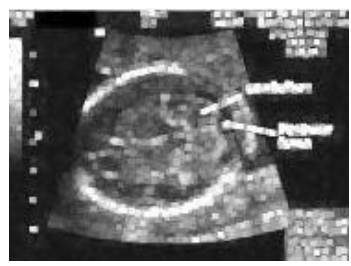

(J)

(A) Original USG image without noise (B) USG Image with speckle noise (C) Alpha trimmed mean filter for speckle noise in USG (D) Arithmetic mean filter for speckle noise in USG (E) Contra harmonic mean filter for speckle noise in USG image (F) Harmonic mean filter for speckle noise in USG image (G) Median filter for speckle noise in USG image (H) Midpoint filter for speckle noise in USG image (I) Minimum filter for speckle noise in USG image ( $\mathbf{J})$ Maximum filter for speckle noise in USG.

\begin{tabular}{|c|c|c|c|c|c|c|c|}
\hline & $\begin{array}{l}\text { Max } \\
\text { diff }\end{array}$ & $\begin{array}{l}\text { NA } \\
\text { E }\end{array}$ & $\begin{array}{l}\mathrm{NC} \\
\mathrm{C}\end{array}$ & $\begin{array}{l}\text { Struc } \\
\text { tural } \\
\text { Cont } \\
\text { ent }\end{array}$ & $\begin{array}{l}\text { Avera } \\
\text { ge } \\
\text { Differ } \\
\text { ence }\end{array}$ & MSE & $\begin{array}{l}\text { PSN } \\
\text { R }\end{array}$ \\
\hline $\begin{array}{l}\text { Alpha } \\
\text { trimm } \\
\text { ed } \\
\text { mean } \\
\text { filter }\end{array}$ & $\begin{array}{l}- \\
24.9 \\
214\end{array}$ & $\begin{array}{l}0.2 \\
564\end{array}$ & $\begin{array}{l}1.2 \\
564\end{array}$ & $\begin{array}{l}1.808 \\
4\end{array}$ & $\begin{array}{l}- \\
24.92 \\
14\end{array}$ & $\begin{array}{l}621.0 \\
756\end{array}$ & $\begin{array}{l}20.1 \\
994\end{array}$ \\
\hline $\begin{array}{l}\text { Arith } \\
\text { metic } \\
\text { mean } \\
\text { filter }\end{array}$ & $\begin{array}{l}- \\
27.8 \\
727\end{array}$ & $\begin{array}{l}0.5 \\
982\end{array}$ & $\begin{array}{l}1.5 \\
982\end{array}$ & $\begin{array}{l}0.391 \\
5\end{array}$ & $\begin{array}{l}- \\
27.87 \\
27\end{array}$ & $\begin{array}{l}776.8 \\
880\end{array}$ & $\begin{array}{l}19.2 \\
272\end{array}$ \\
\hline $\begin{array}{l}\text { Contr } \\
\text { a } \\
\text { harmo } \\
\text { nic } \\
\text { mean } \\
\text { filter }\end{array}$ & $\begin{array}{l}- \\
23.7 \\
897\end{array}$ & $\begin{array}{l}0.5 \\
106\end{array}$ & $\begin{array}{l}1.5 \\
106\end{array}$ & $\begin{array}{l}0.438 \\
2\end{array}$ & $\begin{array}{l}- \\
23.78 \\
97\end{array}$ & $\begin{array}{l}565.9 \\
495\end{array}$ & $\begin{array}{l}20.6 \\
030\end{array}$ \\
\hline $\begin{array}{l}\text { Harm } \\
\text { onic } \\
\text { mean } \\
\text { filter }\end{array}$ & $\begin{array}{l}- \\
32.6 \\
215\end{array}$ & $\begin{array}{l}0.7 \\
001\end{array}$ & $\begin{array}{l}1.7 \\
001\end{array}$ & $\begin{array}{l}0.346 \\
0\end{array}$ & $\begin{array}{l}- \\
32.62 \\
15\end{array}$ & $\begin{array}{l}1.064 \\
2 \\
e+03\end{array}$ & $\begin{array}{l}17.8 \\
607\end{array}$ \\
\hline $\begin{array}{l}\text { Maxi } \\
\text { mum }\end{array}$ & $\begin{array}{l}- \\
18.5\end{array}$ & $\begin{array}{l}0.3 \\
975\end{array}$ & $\begin{array}{l}1.3 \\
975\end{array}$ & $\begin{array}{l}0.152 \\
0\end{array}$ & $\begin{array}{l}- \\
18.52\end{array}$ & $\begin{array}{l}343.0 \\
631\end{array}$ & $\begin{array}{l}22.7 \\
771\end{array}$ \\
\hline
\end{tabular}

\begin{tabular}{|l|l|l|l|l|l|l|l|}
\hline filter & 220 & & & & 20 & & \\
\hline $\begin{array}{l}\text { Media } \\
\mathrm{n}\end{array}$ & - & 0.6 & 1.6 & 0.379 & - & 842.0 & 18.8 \\
filter & 187 & 228 & 228 & 7 & 29.01 & 864 & 772 \\
\hline $\begin{array}{l}\text { Midp } \\
\text { oint } \\
\text { filter }\end{array}$ & -26.7 & 732 & 732 & 0 & 26.70 & 664 & 977 \\
089 & & & & 89 & & \\
\hline $\begin{array}{l}\text { Mini } \\
\text { mum } \\
\text { filter }\end{array}$ & - & 0.8 & 1.8 & 0.301 & - & 1.461 & 16.4 \\
\hline
\end{tabular}

Table 4: Comparison of Statistical parameters for Speckle noise in USG image

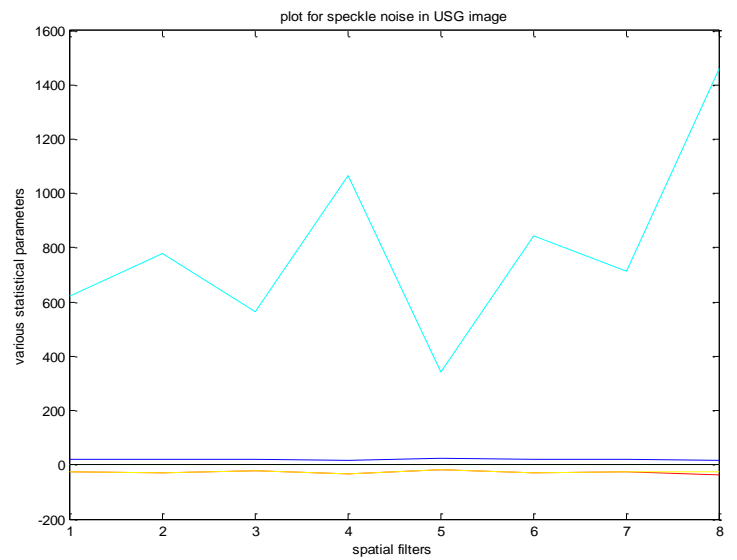

Graph 4: Plot of parameters for Speckle noise in USG image

The results received after comparing all the images of various filters for Gaussian noise in MRI image found Maximum filter as the best filter according to the results obtained.

E. Results for Salt and pepper noise in USG image:

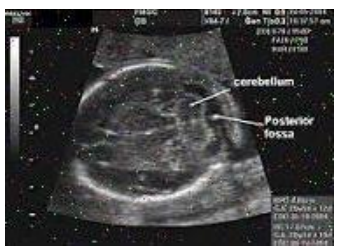

(A)

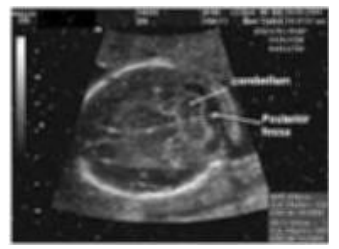

(B) 


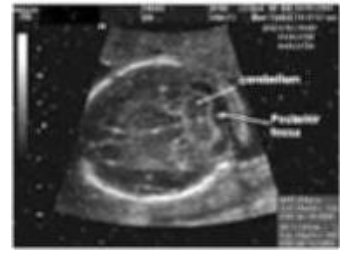

(C)

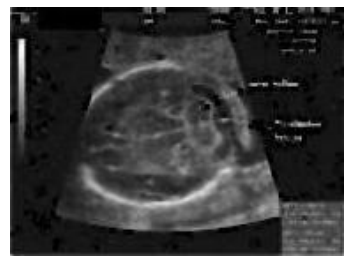

(E)

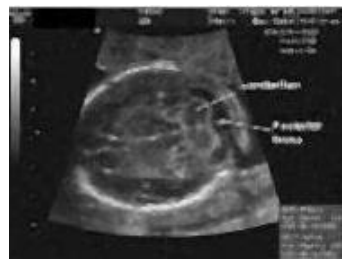

(G)

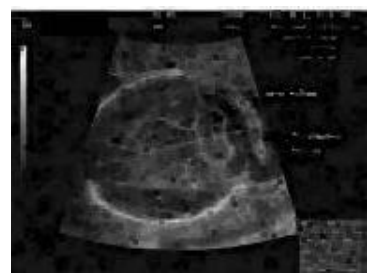

(I)

(A) USG Image with salt and pepper noise (B) Arithmetic mean filter for speckle noise in USG (C) Alpha trimmed mean filter for speckle noise in USG (D) Contra harmonic mean filter for salt and pepper noise in USG (E) Harmonic mean filter for speckle noise in USG (F) Maximum filter for salt and pepper noise in USG (G) Median filter for salt and pepper noise in USG (H) Midpoint filter for salt and pepper noise in USG image (I) Minimum filter for salt and pepper noise in USG.

\begin{tabular}{|l|l|l|l|l|l|l|l|}
\hline & $\begin{array}{l}\text { Max } \\
\text { diff }\end{array}$ & $\begin{array}{l}\text { NA } \\
\text { E }\end{array}$ & $\begin{array}{l}\text { NC } \\
\text { C }\end{array}$ & $\begin{array}{l}\text { Struc } \\
\text { tural } \\
\text { conte } \\
\text { nt }\end{array}$ & $\begin{array}{l}\text { Aver } \\
\text { age } \\
\text { differ } \\
\text { ence }\end{array}$ & MSE & $\begin{array}{l}\text { PSN } \\
\text { R }\end{array}$ \\
\hline $\begin{array}{l}\text { Alpha } \\
\text { trimm } \\
\text { ed } \\
\text { mean }\end{array}$ & - & 0.2 & 1.2 & 1.803 & - & 605. & 20.3 \\
977 & 553 & 553 & 2 & $\begin{array}{l}24.59 \\
77\end{array}$ & 0447 & 129 \\
\hline
\end{tabular}

\begin{tabular}{|c|c|c|c|c|c|c|c|}
\hline filter & & & & & & & \\
\hline $\begin{array}{l}\text { Arith } \\
\text { metic } \\
\text { mean } \\
\text { filter }\end{array}$ & $\begin{array}{l}- \\
27.3 \\
825\end{array}$ & $\begin{array}{l}0.5 \\
877\end{array}$ & $\begin{array}{l}1.5 \\
877\end{array}$ & $\begin{array}{l}0.396 \\
7\end{array}$ & $\begin{array}{l} \\
27.38 \\
25\end{array}$ & $\begin{array}{l}749 . \\
8025\end{array}$ & $\begin{array}{l}19.3 \\
813\end{array}$ \\
\hline $\begin{array}{l}\text { Contr } \\
\text { a } \\
\text { harmo } \\
\text { nic } \\
\text { mean } \\
\text { filter }\end{array}$ & $\begin{array}{l}- \\
23.3 \\
248\end{array}$ & $\begin{array}{l}0.5 \\
006\end{array}$ & $\begin{array}{l}1.5 \\
006\end{array}$ & $\begin{array}{l}0.444 \\
1\end{array}$ & $\begin{array}{l}- \\
23.32 \\
48\end{array}$ & $\begin{array}{l}544 . \\
0481\end{array}$ & $\begin{array}{l}20.7 \\
744\end{array}$ \\
\hline $\begin{array}{l}\text { Harm } \\
\text { onic } \\
\text { mean } \\
\text { filter }\end{array}$ & $\begin{array}{l}- \\
31.8 \\
695\end{array}$ & $\begin{array}{l}0.6 \\
840\end{array}$ & $\begin{array}{l}1.6 \\
840\end{array}$ & $\begin{array}{l}0.352 \\
6\end{array}$ & $\begin{array}{l}- \\
31.86 \\
95\end{array}$ & $\begin{array}{l}1.01 \\
57 \\
\mathrm{e}+03\end{array}$ & $\begin{array}{l}18.0 \\
633\end{array}$ \\
\hline $\begin{array}{l}\text { Maxi } \\
\text { mum } \\
\text { filter }\end{array}$ & $\begin{array}{l}- \\
18.2 \\
838\end{array}$ & $\begin{array}{l}0.3 \\
924\end{array}$ & $\begin{array}{l}1.3 \\
924\end{array}$ & $\begin{array}{l}0.515 \\
8\end{array}$ & $\begin{array}{l}- \\
18.28 \\
38\end{array}$ & $\begin{array}{l}334 . \\
2981\end{array}$ & $\begin{array}{l}22.8 \\
895\end{array}$ \\
\hline $\begin{array}{l}\text { Media } \\
\mathrm{n} \\
\text { filter }\end{array}$ & $\begin{array}{l}- \\
28.4 \\
928\end{array}$ & $\begin{array}{l}0.6 \\
115\end{array}$ & $\begin{array}{l}1.6 \\
115\end{array}$ & $\begin{array}{l}0.385 \\
1\end{array}$ & $\begin{array}{l}- \\
28.49 \\
28\end{array}$ & $\begin{array}{l}811 . \\
8397\end{array}$ & $\begin{array}{l}19.0 \\
361\end{array}$ \\
\hline $\begin{array}{l}\text { Midp } \\
\text { oint } \\
\text { filter }\end{array}$ & $\begin{array}{l}- \\
26.1 \\
414\end{array}$ & $\begin{array}{l}0.5 \\
610\end{array}$ & $\begin{array}{l}1.5 \\
610\end{array}$ & $\begin{array}{l}0.410 \\
4\end{array}$ & $\begin{array}{l}- \\
26.14 \\
14\end{array}$ & $\begin{array}{l}643 . \\
3743\end{array}$ & $\begin{array}{l}19.7 \\
842\end{array}$ \\
\hline $\begin{array}{l}\text { Mini } \\
\text { mum } \\
\text { filter }\end{array}$ & $\begin{array}{l}- \\
36.7 \\
725\end{array}$ & $\begin{array}{l}0.7 \\
892\end{array}$ & $\begin{array}{l}1.7 \\
892\end{array}$ & $\begin{array}{l}0.312 \\
4\end{array}$ & $\begin{array}{l}- \\
36.77 \\
25\end{array}$ & $\begin{array}{l}1.35 \\
22 \\
e+03\end{array}$ & $\begin{array}{l}16.8 \\
203\end{array}$ \\
\hline
\end{tabular}

Table 5: Comparison of Statistical parameters for Salt and pepper noise in USG image 


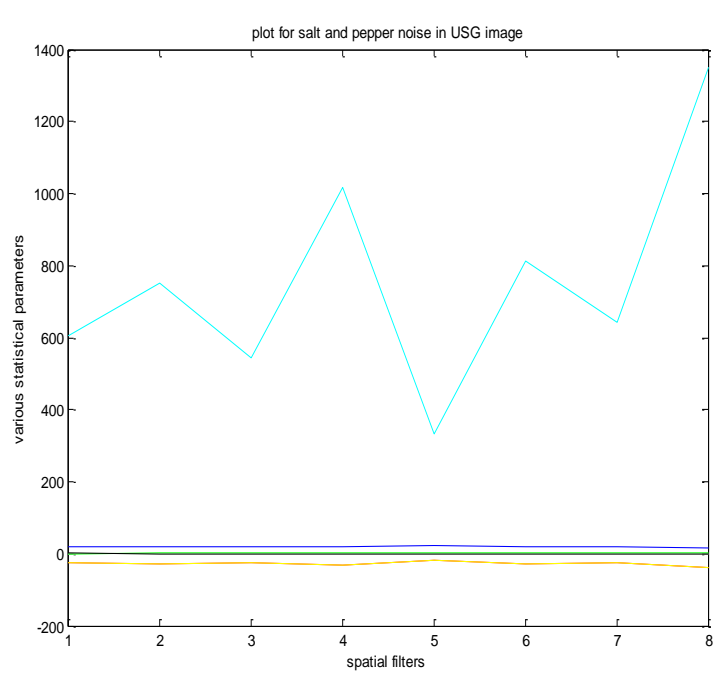

Graph 5: Plot of parameters for Salt and pepper noise in USG image

Alpha trimmed mean filter and maximum filter are the best filters.

F. Results for Gaussian noise in USG image:

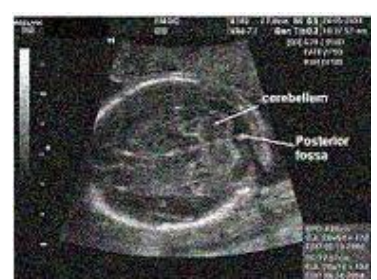

(A)

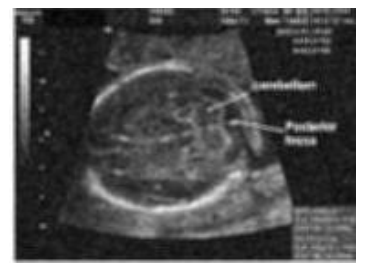

(C)

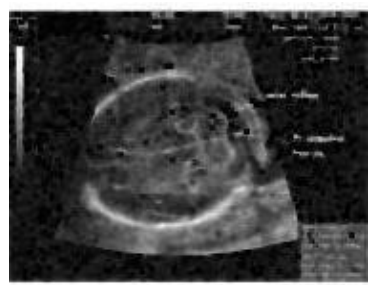

(E)

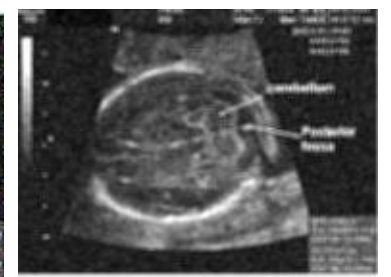

(B)

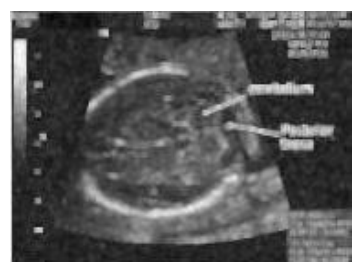

(D)

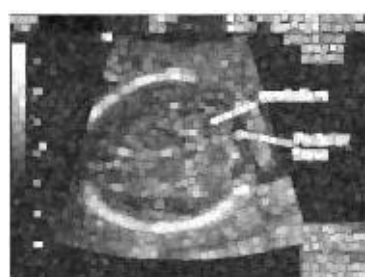

(F)

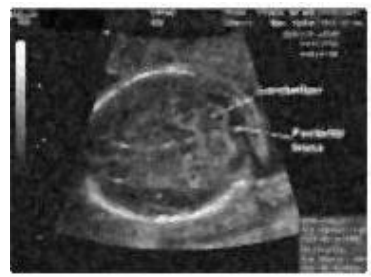

(G)

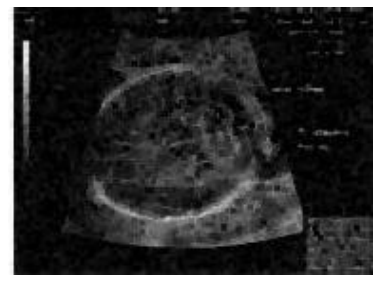

(I)

(A) Image with Gaussian noise (B) Alpha mean filter for Gaussian noise in USG (C) Arithmetic mean filter for Gaussian noise in USG (D) Contra harmonic mean filter for Gaussian noise in USG (E) Harmonic mean filter for Gaussian noise in USG (F) Maximum filter for Gaussian noise in USG image (G) Median filter for Gaussian noise in USG image (H) Midpoint filter for Gaussian noise in USG image (I) Minimum filter for Gaussian noise in USG image.

\begin{tabular}{|l|l|l|l|l|l|l|l|}
\hline & $\begin{array}{l}\text { Max } \\
\text { diff }\end{array}$ & $\begin{array}{l}\text { EA } \\
\text { C }\end{array}$ & $\begin{array}{l}\text { NC } \\
\text { tural } \\
\text { conte } \\
\text { nt }\end{array}$ & $\begin{array}{l}\text { Aver } \\
\text { differ } \\
\text { ence }\end{array}$ & MSE & PSN \\
R
\end{tabular}




\begin{tabular}{|c|c|c|c|c|c|c|c|}
\hline $\begin{array}{l}\text { onic } \\
\text { mean } \\
\text { filter }\end{array}$ & $\begin{array}{l}31.5 \\
692\end{array}$ & 775 & 775 & 3 & $\begin{array}{l}31.56 \\
92\end{array}$ & 6137 & 455 \\
\hline $\begin{array}{l}\text { Maxi } \\
\text { mum } \\
\text { filter }\end{array}$ & $\begin{array}{l}- \\
47.5 \\
398\end{array}$ & $\begin{array}{l}1.0 \\
203\end{array}$ & $\begin{array}{l}2.0 \\
203\end{array}$ & $\begin{array}{l}0.245 \\
0\end{array}$ & $\begin{array}{l}- \\
47.53 \\
98\end{array}$ & $\begin{array}{l}2.26 \\
00 \\
e+03\end{array}$ & $\begin{array}{l}14.5 \\
896\end{array}$ \\
\hline $\begin{array}{l}\text { Media } \\
\mathrm{n} \\
\text { filter }\end{array}$ & $\begin{array}{l}- \\
26.3 \\
753\end{array}$ & $\begin{array}{l}0.5 \\
661\end{array}$ & $\begin{array}{l}1.5 \\
661\end{array}$ & $\begin{array}{l}0.407 \\
7\end{array}$ & $\begin{array}{l}- \\
26.37 \\
53\end{array}$ & $\begin{array}{l}695 . \\
6570\end{array}$ & $\begin{array}{l}19.7 \\
069\end{array}$ \\
\hline $\begin{array}{l}\text { Midp } \\
\text { oint } \\
\text { filter }\end{array}$ & $\begin{array}{l}- \\
24.5 \\
247\end{array}$ & $\begin{array}{l}0.5 \\
264\end{array}$ & $\begin{array}{l}1.5 \\
264\end{array}$ & $\begin{array}{l}0.429 \\
2\end{array}$ & $\begin{array}{l}- \\
24.52 \\
47\end{array}$ & $\begin{array}{l}601 . \\
4624\end{array}$ & $\begin{array}{l}20.3 \\
387\end{array}$ \\
\hline $\begin{array}{l}\text { Mini } \\
\text { mum } \\
\text { filter }\end{array}$ & $\begin{array}{l}- \\
38.3 \\
951\end{array}$ & $\begin{array}{l}0.8 \\
240\end{array}$ & $\begin{array}{l}1.8 \\
240\end{array}$ & $\begin{array}{l}0.300 \\
6\end{array}$ & $\begin{array}{l}- \\
38.39 \\
51\end{array}$ & $\begin{array}{l}1.47 \\
42 \\
e+03\end{array}$ & $\begin{array}{l}16.4 \\
453\end{array}$ \\
\hline
\end{tabular}

Table 6: Comparison of Statistical parameters for Gaussian noise in USG image

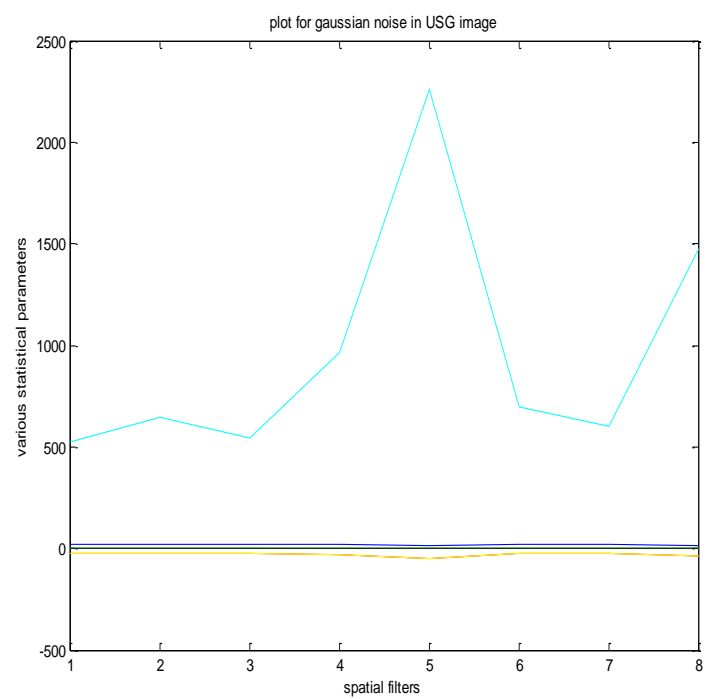

Graph 6: Plot of parameters on Gaussian noise in USG image
Comparing all the images of filters for Speckle noise in USG image and found that Alpha trimmed mean filter and maximum filter are the best filter.

\section{REFERENCES}

[1] R.C. Gonzalez and R.E. Woods, Digital Image Processing, 2nd ed. Englewood Cliffs, NJ: PrenticeHall; 2002.

[2] S. Kent, O. N. Oçan, and T. Ensari. "Speckle Reduction of Synthetic Aperture Radar Images Using Wavelet Filtering". in ITG, VDE, FGAN, DLR, EADS, astrium. EUSAR 2004 Proceedings, 5th European Conference on Synthetic Aperture Radar, May 25-27, 2004, Ulm, Germany.

[3] Charles Boncelet (2005).'Image Noise Models". in Alan C. Bovik. Handbook of Image and Video Processing.

[4] S. Kumar, P. Kumar, M. Gupta and A. K. Nagawat, "Performance Comparison of Median and Wiener Filter in Image De-noising," International Journal of Computer Applications 12(4):27-31, December 2010.

[5] B. Goossens, A. Pizurica, and W. Philips. Image denoising using mixtures of projected Gaussian scale mixtures, IEEE Transactions on Image Processing, Vol.18, Issue. 8, pp.1689-1702, 2009.

[6] R.C. Gonzalez and R.E. Woods, Digital Image Processing, 2nd ed. Englewood Cliffs, NJ: PrenticeHall; 2002.

[7] A.K. Jain, Fundamentals of Digital Image Processing., Englewood Cliffs, NJ: Prentice Hall; 1989.

[8] S. Arivazhagan, S. Deivalakshmi, K. Kannan, B.N.Gajbhiye, C.Muralidhar, Sijo N Lukose, M.P.Subramanian, "Performance Analysis of Wavelet Filters for Image Denoising", Advances in Computational Sciences and Technology, Vol.1 No. 1 (2007), pp. 1-10, ISSN 0973-6107. 